\title{
Band-edge exciton states in AIN single crystals and epitaxial layers
}

\author{
L. Chen and B. J. Skromme ${ }^{\text {a) }}$ \\ Department of Electrical Engineering and Center for Solid State Electronics Research, \\ Arizona State University, Tempe, Arizona 85287-5706 \\ R. F. Dalmau, R. Schlesser, and Z. Sitar \\ Department of Materials Science and Engineering, North Carolina State University, Raleigh, \\ North Carolina 27695-7919 \\ C. Chen, W. Sun, J. Yang, and M. A. Khan \\ Department of Electrical Engineering, University of South Carolina, Columbia, South Carolina 29208 \\ M. L. Nakarmi, J. Y. Lin, and H.-X. Jiang \\ Department of Physics, Kansas State University, Manhattan, Kansas 66506-2601
}

(Received 18 June 2004; accepted 14 September 2004)

\begin{abstract}
The band-edge excitonic properties of AlN are investigated using low-temperature (1.7 K) optical reflectance and transmission measurements of samples with various crystal orientations. The $A, B$, and $C$ excitons are found to have energies of $6.025,6.243$, and $6.257 \mathrm{eV}$ in unstrained material, which shift with strain. The results are compared to a calculation of exciton energies and oscillator strengths to yield a crystal-field splitting of $-230 \mathrm{meV}$ in unstrained AlN, in good agreement with previous ab initio calculations. (C) 2004 American Institute of Physics. [DOI: 10.1063/1.1818733]
\end{abstract}

Aluminum nitride, a very wide band-gap wurtzite IIInitride semiconductor, is of increasing interest as a substrate for both short-wavelength optoelectronic devices and hightemperature high-power electronic devices. It is also an endpoint of the important AlGaN alloy system used in such devices. The optical and excitonic properties of this material have been investigated by several groups. Band-edge luminescence has been investigated using cathodoluminescence (CL) or photoluminescence (PL) measurements on both heteroepitaxial $^{1-5}$ and bulk AlN. ${ }^{5-7}$ The features assigned to $A$ and $B$ free excitons in luminescence are generally in the ranges between $6.023-6.033 \mathrm{eV}$ and $6.036-6.041 \mathrm{eV}$, respectively. $4,6,7$

However, early transmission measurements typically indicated a substantially larger band gap near $6.2 \mathrm{eV}$, and recent low-temperature optical reflectance and ellipsometry studies have assigned excitonic features in the range from $6.216-6.250 \mathrm{eV}$ to $A$ free excitons, and features in the range from $6.268-6.360 \mathrm{eV}$ to $B$ or unresolved $B$ and $C$ free excitons. $^{8-11}$ A large Stokes shift therefore seems to exist between reflectance (absorption) and luminescence peak energies, which is not understood. Moreover, the oscillator strength of the absorption/reflectance feature assigned to $A$ excitons has generally been much larger relative to that of the feature assigned to $B / C$ excitons than would be expected based on polarization selection rules. ${ }^{9-11} \mathrm{~A}$ related issue is the determination of the crystal-field splitting parameter, $\Delta_{\mathrm{cr}},{ }^{12}$ which largely sets the separation between $A$ and $B / C$ excitons. Recent experiments imply values which vary widely from $-0.017 \mathrm{eV}$ (Refs. 6 and 7) to $-0.055 \mathrm{eV}$ (Ref. 9) to $-0.110 \mathrm{eV}$ (Ref. 11).

In the present study, low-temperature $(1.8 \mathrm{~K})$ optical reflectance measurements were performed on a bulk AlN single crystal and on AIN epilayers grown on sapphire substrates to characterize the excitonic properties of AlN. Theo-

\footnotetext{
a) Author to whom correspondence should be addressed; electronic mail: skromme@asu.edu
}

retical calculations of exciton energies and oscillator strengths are discussed and correlated to the experimental results. The results yield a reasonably accurate crystal-field splitting value of $-0.230 \mathrm{eV}$, significantly different from previous experiments. They also clarify the existing confusion in the literature.

An AlN bulk single crystal was grown at a temperature of $1900{ }^{\circ} \mathrm{C}$ in a resistively heated growth reactor, employing sublimation of AlN powder in a nitrogen atmosphere at North Carolina State University (NCSU). ${ }^{13} \mathrm{~A} \sim 0.5 \mu \mathrm{m}$ thick AlN epilayer was grown by low-pressure metal organic chemical vapor deposition (MOCVD) around $1000{ }^{\circ} \mathrm{C}$ on a sapphire substrate at the University of South Carolina (USC). Another $\sim 2 \mu \mathrm{m}$ thick AlN epilayer was grown by MOCVD on a sapphire substrate at Kansas State University (KSU). Low-temperature optical reflectance spectra were measured at $1.7 \mathrm{~K}$ with a high radiance $30 \mathrm{~W}$ deuterium lamp. The light was dispersed by an ISA HR-320 monochromator with a 2400 grooves $/ \mathrm{mm}$ grating, and then incident on the samples at an angle of $\sim 45^{\circ}$. A Hamamatsu R-7154 photomultiplier tube was used to collect the reflected light. Nitrogen purging was used, and all spectra are normalized to the reflectance of a double polished sapphire substrate (absolute reflectivity was not determined).

The bulk AIN crystal has a relatively flat naturally occurring $m$ face, which permits measurements in both $\sigma$ - and $\pi$-configurations. Figure 1 shows reflectance spectra of this sample with its $c$ axis (identified by $\mathrm{x}$-ray Laue diffraction experiments) either parallel or perpendicular to the spectrometer slits (no polarizers were used). Due to the (unknown) partial polarization properties of the spectrometer gratings, the light reflected from the $m$ face contains exciton features from mixed $\sigma$ and $\pi$ configurations, whose relative strengths change with orientation. The theoretical calculations discussed below show that $B$ and $C$ excitons have most of the oscillator strength in $\sigma(\mathbf{E} \perp c)$ configuration while the $A$ exciton dominates in $\pi(\mathbf{E} \| c)$ configuration. We assign the lower-energy excitonic structure at $6.024 \mathrm{eV}$ to the $A$ free 


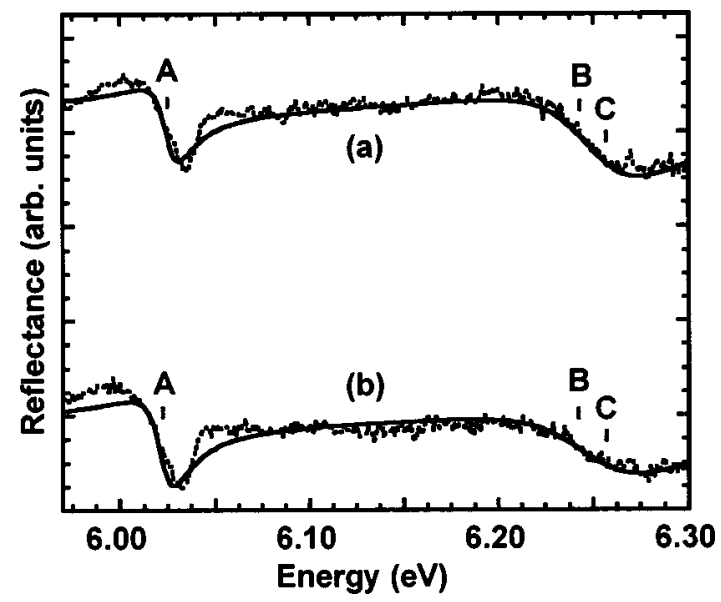

FIG. 1. Low-temperature partially polarized optical reflectance spectra from the $m$ face of a bulk AlN crystal with (a) the $c$ axis parallel to the spectrometer slits and (b) the $c$ axis perpendicular to the slits (dotted lines). Solid lines are theoretical fits to spectra. The bottom of the graph is a (different) finite signal level in each case, to show the features more clearly.

exciton, and the broad structure near $6.25 \mathrm{eV}$ to unresolved free $B$ and $C$ excitons, which are expected to be only $14 \mathrm{meV}$ apart. Previous reflectance measurements generally involved backreflection from $c$ faces ( $\sigma$-polarization), where the $A$ exciton is nearly forbidden and only the $B / C$ exciton structure should be detectable.

The reflectance spectra were fitted using a standard model dielectric function with three Lorentzian oscillators for the $A, B$, and $C$ excitons, including an exciton-free dead layer at the surface. ${ }^{12}$ Spatial dispersion is neglected due to the breadth of the observed features. As the $B$ and $C$ excitons are not resolved and should have nearly identical oscillator strengths, we fixed their separation at the theoretical value of $2 \Delta_{\mathrm{so}} / 3\left(\Delta_{\mathrm{so}}\right.$ is the spin-orbit splitting parameter, ${ }^{12}$ taken to be $20 \mathrm{meV})^{14-17}$ and assumed equal broadening parameters and polarizabilities. The energies and broadening parameters ( $\Gamma$ ) of the three excitons are about 6.025, 6.243, and $6.257 \mathrm{eV}$ and $0.019,0.070$, and $0.070 \mathrm{eV}$, respectively; the dead layer thickness is around $3.3 \mathrm{~nm}$. The polarizabilities $(4 \pi \alpha)$ of the $B$ and $C$ excitons decrease from 0.0062 to 0.0051 when the sample is rotated, while the polarizability of the $A$ exciton increases from 0.0033 to 0.0043 . This behavior is consistent with the theoretically expected oscillator strengths in the two different configurations. Preliminary measurements using a Rochon polarizer and a half-wave plate to achieve purer polarizations (not shown) show that the $A$ exciton feature is clearly observed and the $B / C$ excitons are undetectable (for our signal-to-noise ratio) in $\pi$-polarization, whereas the $A$ exciton is about two times weaker and the $B / C$ exciton feature is clearly present in $\sigma$ polarization. The residual $A$ exciton feature in $\sigma$-polarization may be due to imperfect polarization optics. The $B$ and $C$ excitons are broader than the $A$ exciton, in spite of their lower sensitivity to inhomogeneous strains. We believe this is because they are degenerate with the continuum (unbound states) of the $A$ excitons and therefore experience greater scattering. We have observed similar anomalous broadening for the $C$ exciton in $\mathrm{GaN}$ when strain causes it to become degenerate with the continuum of the $A$ exciton.

Figure 2 shows reflectance spectra of the MOCVD AlN/ sapphire (0001) layers along with theoretical fits similar to those described above; the finite thicknesses of the epilayers

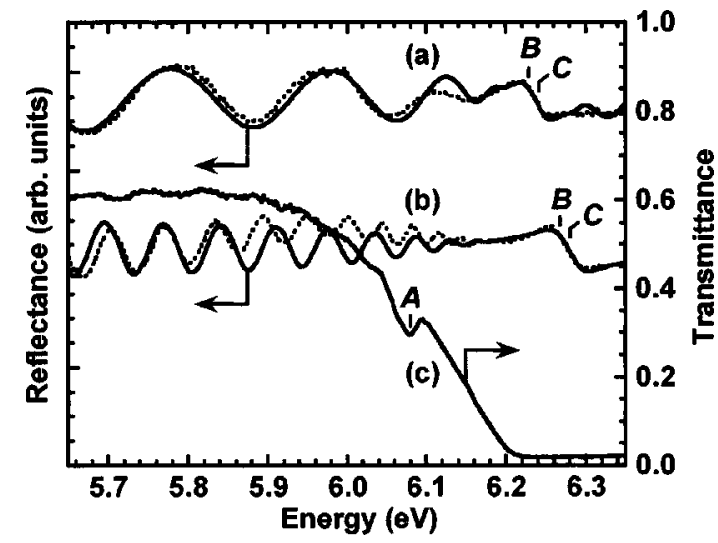

FIG. 2. Low-temperature optical reflectance spectra of the MOCVD AIN/ sapphire (0001) layers of (a) $\sim 0.5 \mu \mathrm{m}$ and (b) $\sim 2 \mu \mathrm{m}$ thicknesses (dotted lines). Solid lines are fits, yielding $E_{B}=6.228 \mathrm{eV}, E_{C}=6.242 \mathrm{eV}$, and $\Gamma_{B} / \Gamma_{C}=0.028 \mathrm{eV}$ for the $0.5 \mu \mathrm{m}$ layer, and $E_{B}=6.267 \mathrm{eV}, E_{C}=6.281 \mathrm{eV}$, and $\Gamma_{B} / \Gamma_{C}=0.043 \mathrm{eV}$ for the $2 \mu \mathrm{m}$ layer. A constant background AlN dielectric constant of $8.2+0.12 i$ or $8.2+0.045 i$ was used in each case, respectively, and layer thicknesses were adjusted. (c) Transmission spectrum of the $2 \mu \mathrm{m}$ layer of part (b). The signal above $\sim 6.2 \mathrm{eV}$ is due to stray light and dark current.

and the optical properties of the sapphire substrates were included in the model. A transmission spectrum of the thicker layer is also shown. Structures assigned to unresolved $B$ and $C$ excitons are observed near 6.238 and $6.275 \mathrm{eV}$ in the 0.5 and $2.0 \mu \mathrm{m}$ thick layers, respectively. The FabryPerot interference fringes below the AlN band gap reflect the layer thicknesses; they are not modeled perfectly because of dispersion in the AIN refractive index due to band-to-band absorptions (not included). The energy shifts of these spectra, relative to that of the bulk sample, are attributed to biaxial compressive and tensile strains in the thicker and thinner layers, respectively. Experimental measurements are in progress to confirm those strains. The $A$ exciton is much too weak to be detected in either of the necessarily purely $\sigma$-polarized reflectance spectra (especially in the presence of interference fringes). However, a weak dip is clearly observed in the transmission spectrum of the thicker layer at $6.080 \mathrm{eV}$, which we assign to an $A$ free-exciton state shifted up in energy from its strain-free position by biaxial compressive strain. It is clearly separated from the much stronger absorption edge due to the $B$ and $C$ excitons above $6.2 \mathrm{eV}$.

Using the Rashba-Sheka-Pikus Hamiltonian and the usual Hopfield quasi-cubic approximation, ${ }^{12}$ we calculated the exciton energies and oscillator strengths in wurtzite AlN. Large negative values of $\Delta_{\mathrm{cr}}$, ranging from $-169 \mathrm{meV}$ to $-244 \mathrm{meV}$, were predicted in various $a b$ initio calculations. ${ }^{4,14-20}$ (Suzuki et al. ${ }^{17}$ obtained a smaller value of $-58.5 \mathrm{meV}$ but did not include atomic relaxations, so this value is likely to be inaccurate.) All existing calculations of $\Delta_{\text {so }}$ predict values within $1 \mathrm{meV}$ of $20 \mathrm{meV} .{ }^{14-17}$ Given that $\Delta_{\text {so }} \ll\left|\Delta_{\text {cr }}\right|$ in AlN, the energies and oscillator strengths are well approximated by the simplified expressions in Table I. The energies are plotted as a function of $\Delta_{\mathrm{cr}}$ in Fig. 3, where the various theoretical predictions of $\Delta_{\mathrm{cr}}$ are also indicated. The energy of the $A$ exciton is independent of $\Delta_{\mathrm{cr}}$, but those of the $B$ and $C$ excitons vary significantly. The oscillator strengths vary insignificantly, with the $A$ exciton having an oscillator strength less than $0.04 \%$ of the nearly identical strengths of the $B$ and $C$ excitons in $\sigma$-polarization for license or copyright; see http://apl.aip.org/about/rights_and_permissions 
TABLE I. Approximate energies and oscillator strengths of the $A, B$, and $C$ excitons in unstrained wurtzite AlN, assuming that $\left|\Delta_{\text {so }}\right| \ll\left|\Delta_{\text {cr }}\right|$. (Here, $E_{g x}$ is the excitonic band gap in the absence of crystal-field or spin-orbit splittings; and $P_{\perp}$ and $P_{\|}$are the momentum matrix elements for $\sigma$ - and $\pi$-polarizations, respectively.)

\begin{tabular}{lccc}
\hline \hline & & \multicolumn{2}{c}{ Oscillator strengths } \\
\cline { 3 - 4 } Exciton & Energy & $\pi(\mathbf{E} \| c)$ & $\sigma(\mathrm{E} \perp c)$ \\
\hline $\boldsymbol{A}\left(\Gamma_{7}\right)$ & $E_{g x}$ & $2\left|P_{\|}\right|^{2}$ & $\left(2 \Delta_{\mathrm{so}}^{2} / 9 \Delta_{\mathrm{cr}}^{2}\right)\left|P_{\perp}\right|^{2}$ \\
$\boldsymbol{B}\left(\Gamma_{9}\right)$ & $E_{g x}-\Delta_{\mathrm{cr}}-\Delta_{\mathrm{so}} / 3$ & 0 & $\left|P_{\perp}\right|^{2}$ \\
$\boldsymbol{C}\left(\Gamma_{7}\right)$ & $E_{g x}-\Delta_{\mathrm{cr}}+\Delta_{\mathrm{so}} / 3$ & $\left(4 \Delta_{\mathrm{so}}^{2} / 9 \Delta_{\mathrm{cr}}^{2}\right)\left|P_{\|}\right|^{2}$ & $\left|P_{\perp}\right|^{2}$ \\
\hline \hline
\end{tabular}

$\Delta_{\mathrm{cr}}<-0.15 \mathrm{eV}$. The $A$ exciton is completely dominant over the $B$ and $C$ excitons in $\pi$-polarization.

Using the exciton energies of the unstrained bulk sample of Fig. 1 together with the plot in Fig. 3, we find $\Delta_{\mathrm{cr}}=-230 \mathrm{meV}$ in wurtzite AlN. This value is close to the average of the theoretical calculations that included atomic relaxations, -207 meV. ${ }^{4,14-16,18-20}$ Using the effective masses computed by Kim et al. ${ }^{16}$ and a relative static dielectric constant of 8.0, we estimate the exciton binding energy to be $71 \mathrm{meV}$. The band-gap energy of unstrained wurtzite AlN is therefore about $6.096 \mathrm{eV}$ at $1.7 \mathrm{~K}$, close to a value of $6.12 \mathrm{eV}$ estimated from PL measurements. ${ }^{4}$

We conclude that a recent assignment of CL peaks only $\sim 13 \mathrm{meV}$ apart to $A$ and $B$ free excitons (which would imply $\Delta_{\mathrm{cr}}=-0.017 \mathrm{eV}$ ) is incorrect. ${ }^{6,7}$ All observed peaks presumably involved free or bound $A$ excitons, which can be important in luminescence in spite of their low oscillator strengths due to thermalization effects. Also, features assigned to $A$ excitons in recent reflectance and ellipsometry studies would be better assigned to unresolved $B$ and $C$ excitons, which would explain their strengths in light of the selection rule forbidding the $A$ exciton absorption. ${ }^{8,10,11}$ Reference 9 used a fitting procedure that allowed the $A$ and $B / C$ exciton structures to have very different phases, which is inconsistent

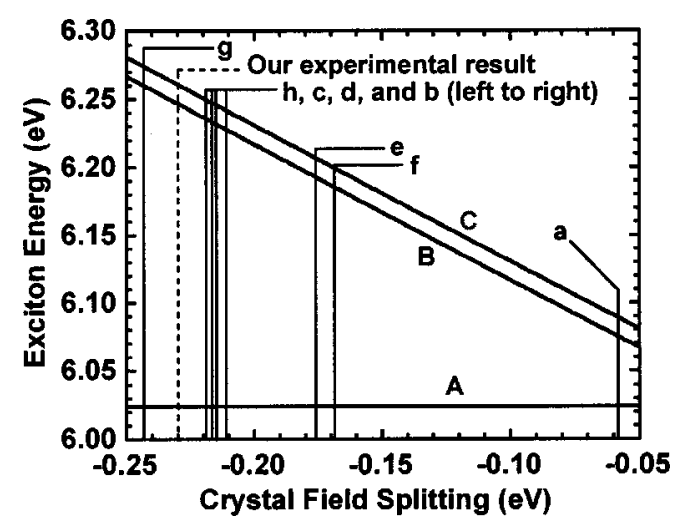

FIG. 3. Energies of the $A, B$, and $C$ excitons in wurtzite AlN as a function of $\Delta_{\mathrm{cr}}$, predicted from the formulas in Table I. We assumed $\Delta_{\mathrm{so}}=20 \mathrm{meV}$ and adjusted $E_{g x}$ to match our experimental data on the bulk sample (dashed line). Various predictions of $\Delta_{\mathrm{cr}}$ are indicated, based on the calculations of (a) [Ref. 17]; (b) [Ref. 14]; (c) [Ref. 15]; (d) [Ref. 16]; (e) [Ref. 18]; (f) [Ref. 19]; (g) [Ref. 20]; and (h) [Ref. 4]. with the usual dead layer model and with prior studies on other materials. We suggest that the feature they observed should be assigned to unresolved $B / C$ excitons only, which explains the anomalous oscillator strengths and $\Delta_{\mathrm{cr}}$ value $(-0.055 \mathrm{eV})$ they found. The anomalously large Stokes shift between absorption/reflectance and luminescence data in the literature is now clearly explained by the oscillator strengths of the different states and the effects of thermalization.

In summary, the $A$ exciton feature of AlN is directly observed in optical reflectance and transmission, which helps to clarify the interpretation of absorption and luminescence properties of this material and to establish a reliable value of $\Delta_{\mathrm{cr}}$. The results imply that high $\mathrm{Al}$ content, (0001)-oriented AlGaN alloys will be much better edge emitters than surface emitters, and other orientations should be considered for surface-emitting devices.

The work at Arizona State University and NCSU was supported by the Office of Naval Research Multidisciplinary University Research Initiative on III-Nitride Crystal Growth and Wafering, Grant No. N00014-01-1-0716, monitored by Dr. C. E. C. Wood. The work at KSU was supported by NSF (DMR-0203373) and DOE (Grant No. DEFG0396ER45604 A005). The authors thank T. L. Groy for performing and analyzing the Laue x-ray diffraction experiments.

${ }^{1}$ X. Tang, F. Hossain, K. Wongchotigul, and M. G. Spencer, Appl. Phys. Lett. 72, 1501 (1998).

${ }^{2}$ N. Teofilov, K. Thonke, R. Sauer, D. G. Ebling, L. Kirste, and K. W. Benz, Diamond Relat. Mater. 10, 1300 (2001).

${ }^{3}$ Y. Shishkin, R. P. Devaty, W. J. Choyke, F. Yun, T. King, and H. Morkoc, Phys. Status Solidi A 188, 591 (2001).

${ }^{4}$ J. Li, K. B. Nam, M. L. Nakarmi, J. Y. Lin, H. X. Jiang, P. Carrier, and S. Wei, Appl. Phys. Lett. 83, 5163 (2003).

${ }^{5}$ E. Kuokstis, J. Zhang, Q. Fareed, J. W. Yang, G. Simin, M. A. Khan, R. Gaska, M. Shur, C. Rojo, and L. Schowalter, Appl. Phys. Lett. 81, 2755 (2002).

${ }^{6}$ J. A. Freitas, Jr., G. C. B. Braga, E. Silveira, J. G. Tischler, and M. Fatemi, Appl. Phys. Lett. 83, 2584 (2003).

${ }^{7}$ E. Silveira, J. A. Freitas, Jr., G. A. Slack, and L. J. Schowalter, Phys. Status Solidi C 0, 2618 (2003); E. Silveira, J. A. Freitas, Jr., M. Kneissl, D. W. Treat, N. M. Johnson, G. A. Slack, and L. J. Schowalter, Appl. Phys. Lett. 84, 3501 (2004).

${ }^{8}$ Q. Guo, M. Nishio, H. Ogawa, and A. Yoshida, Phys. Rev. B 64, 113105 (2001).

${ }^{9}$ T. Onuma, S. F. Chichibu, T. Sota, K. Asai, S. Sumiya, T. Shibata, and M. Tanaka, Appl. Phys. Lett. 81, 652 (2002).

${ }^{10}$ T. Wethkamp, K. Wilmers, C. Cobet, N. Esser, W. Richter, O. Ambacher, M. Stutzmann, and M. Cardona, Phys. Rev. B 59, 1845 (1999).

${ }^{11}$ J. Chen, W. Z. Shen, H. Ogawa, and Q. X. Guo, Appl. Phys. Lett. 84, 4866 (2004).

${ }^{12}$ B. J. Skromme, Mater. Sci. Eng., B 50, 117 (1997), and references therein.

${ }^{13}$ R. Schlesser, R. Dalmau, and Z. Sitar, J. Cryst. Growth 241, 416 (2002).

${ }^{14}$ J. Majewski, M. Stadele, and P. Vogl, MRS Internet J. Nitride Semicond. Res. 1, 30 (1996).

${ }^{15}$ S.-H. Wei and A. Zunger, J. Appl. Phys. 69, 2719 (1996).

${ }^{16}$ K. Kim, W. R. L. Lambrecht, and B. Segall, Phys. Rev. B 56, 7363 (1997).

${ }^{17}$ M. Suzuki, T. Uenoyama, and A. Yanase, J. Appl. Phys. 80, 6868 (1996).

${ }^{18}$ K. Shimada, T. Sota, and K. Suzuki, J. Appl. Phys. 84, 4951 (1998).

${ }^{19}$ S. K. Pugh, D. J. Dugdale, S. Brand, and R. A. Abram, Semicond. Sci. Technol. 14, 23 (1999).

${ }^{20}$ J.-M. Wagner and F. Bechstedt, Phys. Rev. B 66, 115202 (2002). 